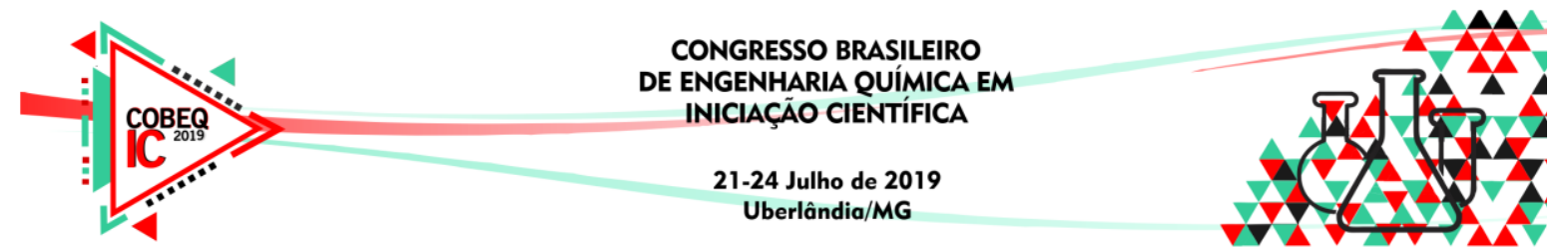

\title{
ESTUDO DO EQUILÍBRIO LÍQUIDO-LÍQUIDO DE SISTEMAS AQUOSOS BIFÁSICOS COMPOSTOS POR 2- PROPANOL + SAIS DE SÓDIO + ÁGUA
}

\author{
I. B. JULIÃO, P. P. A. SOUZA e M. F. MENDES \\ Universidade Federal Rural do Rio de Janeiro, Departamento de Engenharia Química \\ E-mail para contato: marisamf@ufrrj.br
}

\begin{abstract}
RESUMO - O equilíbrio líquido-líquido ternário dos sistemas constituídos por 2propanol + sulfato/nitrato/acetato de sódio + água foram mensurados a 298,15 K e $101,3 \mathrm{kPa}$. Os pontos binodais obtidos experimentalmente foram bem ajustados pelos modelos propostos por Mistry et al. e Merchuk et al. e se mostraram semelhantes aos encontrados na literatura. Através das equações de Othmer-Tobias e Bancroft, concluiu-se que os dados das linhas de amarração eram confiáveis devido aos baixos desvios padrões encontrados. Os sistemas com sulfato apresentaram duas fases muito aquosas, enquanto que os sistemas com nitrato e acetato originaram uma fase superior rica no álcool e uma fase inferior rica em sal e água. Este comportamento é interessante para uma separação entre o 2-propanol e a água usando uma extração líquido-líquido.
\end{abstract}

\section{INTRODUÇÃO}

O 2-propanol, também conhecido como álcool isopropílico, é um composto muito importante para a indústria devido sua versatilidade de utilização (Dutia, 2012). Porém, a desidratação do álcool é difícil devido ao comportamento azeotrópico observado nas soluções aquosas de 2-propanol. Assim, se faz necessário o estudo de processos alternativos de separação que consigam obter este álcool com níveis elevados de pureza. Dentre estas técnicas, a que apresenta o melhor custo benefício é a extração líquido-líquido, sendo que a utilização de solventes orgânicos ou de custo elevado impossibilita sua larga aplicação. Neste cenário, surge a extração líquido-líquido realizada a partir de um sistema aquoso bifásico (SAB) que apresenta várias vantagens, como o menor custo, baixa toxicidade e a possibilidade de aplicação em grande escala (Sanglard et al., 2018).

O funcionamento destes sistemas consiste na separação das fases através do efeito saltingout produzido pelos sais inorgânicos. Pensando na aplicação de um SAB visando um processo extrativo, este trabalho teve como objetivo o estudo do equilíbrio líquido-líquido deste tipo de sistema ternário compostos por 2-propanol + sais de sódio + água a 298,15 K.

\section{MATERIAIS E MÉTODOS}

\subsection{Materiais}




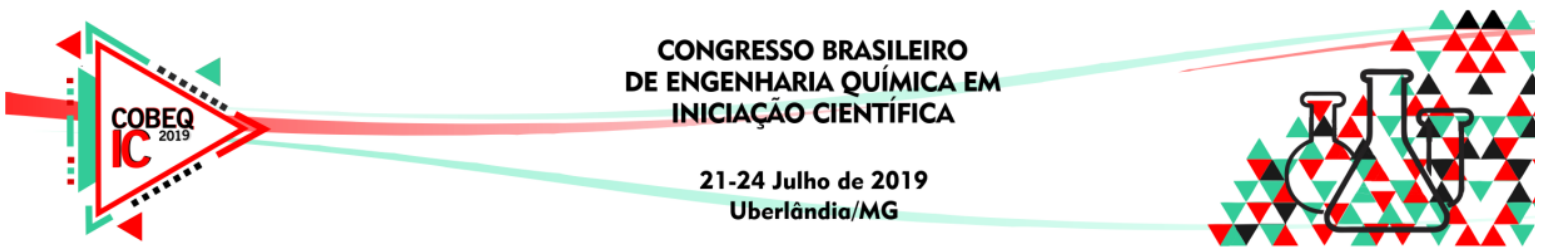

Para a realização dos experimentos utilizou-se os seguintes reagentes químicos: álcool isopropílico P.A. (com 99,5\% de pureza mínima) fornecido pela Proquímios Comércio e Indústria Ltda. (Rio de Janeiro, Brasil); nitrato de sódio P.A. (com 98\% de pureza mínima) adquirido junto a Isofar Indústria e Comércio de Produtos Químicos Ltda (Duque de Caxias, Brasil); sulfato de sódio PA (com 99\% de pureza mínima) e acetato de sódio P.A. (com 99\% de pureza mínima) obtidos através da Vetec Química Fina Ltda. (Rio de Janeiro, Brasil). Todos estes produtos químicos foram utilizados sem nenhuma purificação adicional. Além dos reagentes citados, utilizou-se água destilada em todos os experimentos.

\subsection{Determinação dos dados de equilíbrio líquido-líquido}

A determinação das curvas binodais foi realizada utilizando o método de titulação turbidimétrica (Pimentel et al., 2017) à pressão atmosférica. Foram utilizados o 2-propanol e soluções salinas de $\mathrm{Na}_{2} \mathrm{SO}_{4}$ (30\% em peso), $\mathrm{NaCH}_{3} \mathrm{COO}$ (30\% em peso) e $\mathrm{NaNO}_{3}(45 \%$ em peso) na preparação de SAB. Primeiramente, adicionou-se $3 \mathrm{~g}$ de solução salina em um tubo de ensaio de $20 \mathrm{~mL}$. Posteriormente, o tubo com a solução foi posto em um banho térmico a 298,15 $\mathrm{K}$, durante 30 min para o alcance do equilíbrio térmico. A curva binodal pode ser construída a partir do gotejamento de álcool na solução, sob agitação manual, até o surgimento de uma mistura túrbida, o que indicaria a formação de duas fases. Ao observar o aparecimento da turbidez, a solução era pesada, para medir a quantidade de álcool adicionado, e a composição do ponto binodal.

De forma seguinte, certa quantidade de água destilada foi adicionada até a solução se tornar homogênea. Seguidamente, pesava-se a quantidade de água adicionada e o método era reiniciado para que outro ponto binodal fosse obtido. Este procedimento, feito em triplicata, foi repetido até se obter pontos experimentais suficientes para a plotagem da curva binodal. Estes dados binodais experimentais foram ajustados pelos modelos propostos por Mistry et al. (1996), Equação 1, e Merchuk et al. (1998), Equação 2, sendo w1 e w2 as frações mássicas de 2-propanol e sal, respectivamente, enquanto a, b, c e d são os parâmetros ajustáveis.

$$
\begin{aligned}
& \mathrm{w}_{1}=\exp \left(\mathrm{a}+\mathrm{bw}_{2}^{0,5}+\mathrm{cw}_{2}+\mathrm{dw}_{2}^{2}\right) \\
& \mathrm{w}_{1}=\mathrm{a} \exp \left(-\mathrm{bw}_{2}^{0,5}-\mathrm{cw}_{2}^{3}\right)
\end{aligned}
$$

O método gravimétrico descrito por Merchuk et al. (1998) foi utilizado na obtenção das linhas de amarração. Definiu-se três composições globais e adicionou-se $40 \mathrm{~g}$ da solução ternária escolhida em um becker de $50 \mathrm{~mL}$. Em seguida, a mistura foi agitada durante $1 \mathrm{~h}$, e deixada em repouso por $20 \mathrm{~h}$, em um banho térmico a 298,15 K. O equilíbrio termodinâmico foi considerado atingido quando as fases estavam totalmente límpidas. Para a determinação de tais tempos de repouso e agitação, foi realizado um levantamento bibliográfico e escolhido as condições que melhor atendiam o sistema e as condições de trabalho. Ao atingir o equilíbrio, coletou-se alíquotas de ambas as fases. As amostras retiradas foram posteriormente diluídas para que a concentração de sal fosse determinada pela medida de condutividade elétrica (Lo et al. 2015). A concentração de álcool foi estimada segundo a metodologia proposta por Pimentel et al. (2017), composto pela Regra da Alavanca (Equação 4) e pelas Equações 5 e 6: 


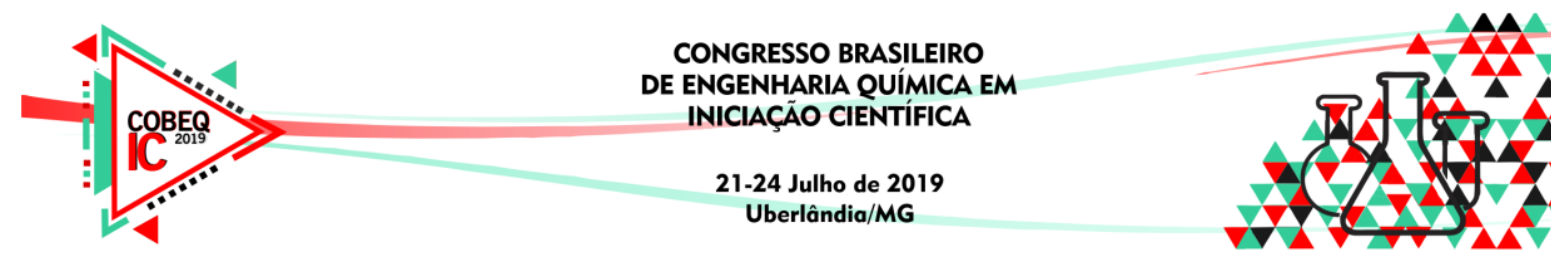

$$
\begin{aligned}
& \frac{\mathrm{w}_{2}-\mathrm{w}_{2}^{\mathrm{s}}}{\mathrm{w}_{2}^{\mathrm{i}}-\mathrm{w}_{2}}=\frac{\mathrm{w}_{1}^{\mathrm{s}}-\mathrm{w}_{1}}{\mathrm{w}_{1}-\mathrm{w}_{1}^{\mathrm{i}}} \\
& \mathrm{w}_{1}^{\mathrm{s}}=\mathrm{f}\left(\mathrm{w}_{2}^{\mathrm{s}}\right) \\
& \mathrm{w}_{1}^{\mathrm{i}}=\mathrm{f}\left(\mathrm{w}_{2}^{\mathrm{i}}\right)
\end{aligned}
$$

sendo os sobrescritos s e i as fases superior e inferior, respectivamente. As frações mássicas de 2-propanol e sal, $\mathrm{w}_{1}$ e $\mathrm{w}_{2}$ representam a composição global da mistura. Todos os experimentos das linhas de amarração foram realizados em triplicata. O comprimento das linhas de amarração (CLA) e a inclinação das linhas de empate (ILA) foram calculados usando Equações 7 e 8.

$$
\begin{aligned}
& \mathrm{CLA}=\left[\left(\mathrm{w}_{1}^{\mathrm{s}}-\mathrm{w}_{1}^{\mathrm{i}}\right)^{2}+\left(\mathrm{w}_{2}^{\mathrm{s}}-\mathrm{w}_{2}^{\mathrm{i}}\right)^{2}\right]^{0,5} \\
& \mathrm{ILA}=\left(\frac{\mathrm{w}_{1}^{\mathrm{s}}-\mathrm{w}_{1}^{\mathrm{i}}}{\mathrm{w}_{2}^{\mathrm{s}}-\mathrm{w}_{2}^{\mathrm{i}}}\right)
\end{aligned}
$$

Para avaliar a precisão dos dados das linhas de amarração, obtidos experimentalmente, foram utilizadas as equações de Othmer-Tobias (1942), Equação 9, e Bancroft (González-Tello, 1996), Equação 10.

$$
\begin{aligned}
& \left(\frac{1-\mathrm{w}_{1}^{\mathrm{s}}}{\mathrm{w}_{1}^{\mathrm{i}}}\right)=\mathrm{k}_{1}\left(\frac{1-\mathrm{w}_{2}^{\mathrm{s}}}{\mathrm{w}_{2}^{\mathrm{i}}}\right)^{\mathrm{n}} \\
& \frac{\mathrm{w}_{3}^{\mathrm{s}}}{\mathrm{w}_{2}^{\mathrm{s}}}=\mathrm{k}_{2}\left(\frac{\mathrm{w}_{3}^{\mathrm{i}}}{\mathrm{w}_{1}^{\mathrm{i}}}\right)^{\mathrm{r}}
\end{aligned}
$$

Sendo $\mathrm{w}_{1}{ }^{\mathrm{s}}, \mathrm{w}_{1}{ }^{\mathrm{i}}, \mathrm{w}_{2}{ }^{\mathrm{s}}, \mathrm{w}_{2}{ }^{\mathrm{i}}, \mathrm{w}_{3}{ }^{\mathrm{s}}$ e $\mathrm{w}_{3}{ }^{\mathrm{i}}$ as frações mássicas de 2-propanol (1), sal (2) e água (3) nas fases superior (s) e inferior (i), respectivamente e $\mathrm{k}_{1}, \mathrm{k}_{2}, \mathrm{n}$ e $\mathrm{r}$ os parâmetros ajustáveis.

\section{RESULTADOS E DISCUSSÃO}

Os dados binodais experimentais foram ajustados pelos modelos de Mistry et al. (1996) e Merchuk et al. (1998) e suas respectivas curvas binodais são mostradas nas Figuras 1B-1D. Os valores de $\mathrm{R}^{2}$ (coeficiente de determinação) muito próximos ou iguais a 1 e os baixos valores de DP (desvios padrões), indicados na Tabela 1, confirmam a boa correlação entre o modelo adotado e os dados experimentais dos sistemas ternários investigados.

Tabela 1 - Parâmetros das Equações 1 e 2 para os sistemas 2-propanol/sais de sódio/água 


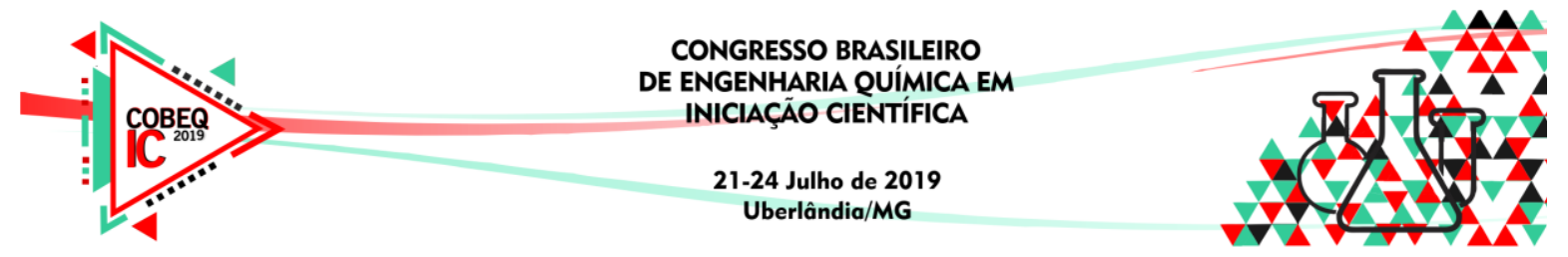

\begin{tabular}{|c|c|c|c|c|c|c|c|}
\hline \multirow{2}{*}{ Sistema } & \multirow{2}{*}{$\begin{array}{l}\text { Melhor } \\
\text { modelo }\end{array}$} & \multicolumn{6}{|c|}{ Parâmetros } \\
\hline & & $\mathrm{a}$ & $\mathrm{b}$ & $\mathrm{c}$ & $\mathrm{d}$ & $\mathrm{R}^{2}$ & $\mathrm{DP}$ \\
\hline 2-Propanol/ $\mathrm{Na}_{2} \mathrm{SO}_{4} /$ Água & Mistry & $-0,2151$ & $-5,1325$ & 3,8450 & $-26,6878$ & 1,0000 & 0,0003 \\
\hline 2-Propanol/ $\mathrm{NaCH}_{3} \mathrm{COO} /$ Água & Merchuk & 1,5582 & 3,1632 & 39,5026 & - & 1,0000 & 0,0003 \\
\hline 2-Propanol/ $\mathrm{NaNO}_{3} /$ Água & Merchuk & 1,2074 & 2,4632 & 36,9128 & - & 0,9999 & 0,0009 \\
\hline
\end{tabular}

Figura 1 - Diagramas de equilíbrio líquido-líquido dos sistemas (A) 2-propanol/sal/água; (B)

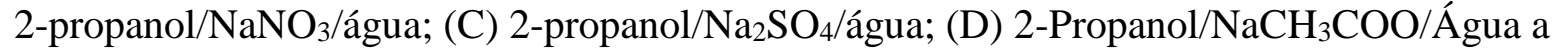

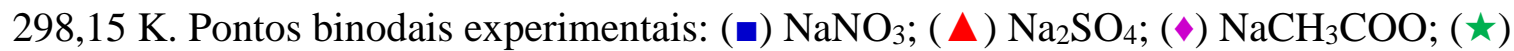
Pimentel et al., 2017; ( $\nabla$ ) Lo et al., 2015. Curva binodal modelada empiricamente: (一) $\mathrm{NaNO}_{3}$; (一) $\mathrm{Na}_{2} \mathrm{SO}_{4}$; (-) $\mathrm{NaCH}_{3} \mathrm{COO}$. (•) Composições experimentais das fases e (†) composições globais das (-) linhas de amarração.
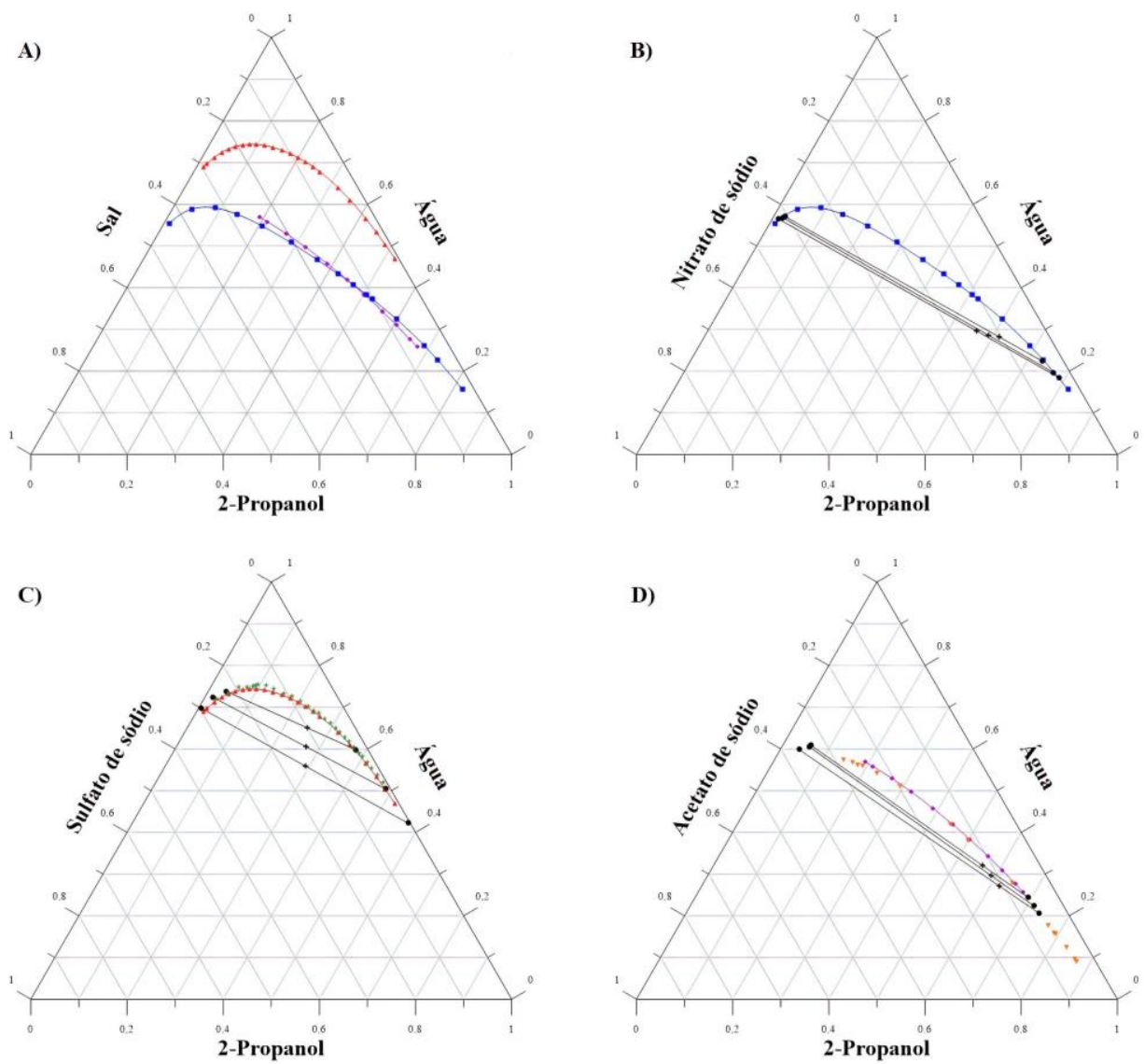

A Figura 1C e 1D mostram certa semelhança entre o comportamento dos dados da literatura e os obtidos neste trabalho. Não foram encontrados dados para sistemas contendo nitrato de sódio e 2-propanol a serem comparados. Os dados experimentais das linhas de amarração estão listados na Tabela 2 e suas representações gráficas são mostradas nas Figuras 1B-1D. Como mostrado na Figuras 1B, as fases superiores foram compostas em grande parte de álcool, enquanto nas fases inferiores, o sal foi predominante, sendo a água o principal componente de ambas as fases. Por outro lado, nas Figuras 1C e 1D observou-se uma fase superior composta principalmente por álcool e uma fase inferior constituída basicamente por sal e água. $\mathrm{O}$ aumento da quantidade de álcool na fase superior, reduziu a concentração de água nessa fase devido a sua migração para fase inferior. 


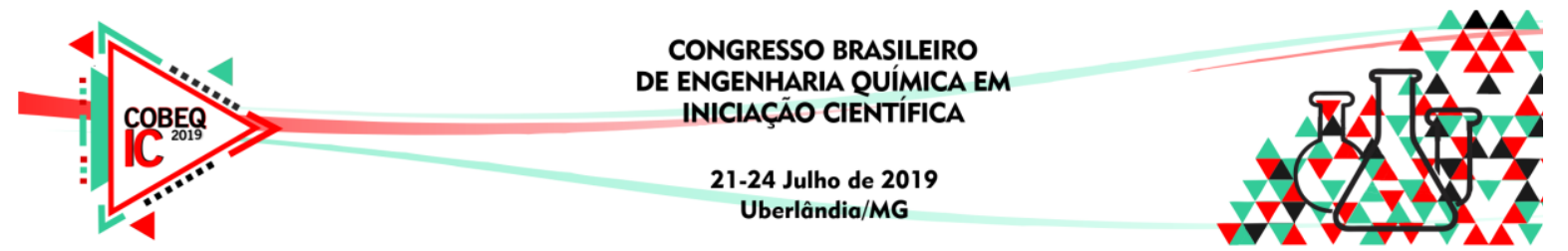

Tabela 2 - Dados experimentais das linhas de amarração para os sistemas 2-propanol/sais de sódio/água a $298,15 \mathrm{~K}$ e $101,3 \mathrm{kPa}$

\begin{tabular}{|c|c|c|c|c|c|c|c|}
\hline \multicolumn{2}{|c|}{ Composições globais } & \multicolumn{2}{|c|}{ Fase Superior } & \multicolumn{2}{|c|}{ Fase Inferior } & \multirow{2}{*}{ CLA } & \multirow{2}{*}{ ILA } \\
\hline $\mathrm{w}_{1}$ & $\mathrm{w}_{2}$ & $\mathrm{w}_{1}$ & $\mathrm{w}_{2}$ & $\mathrm{w}_{1}$ & $\mathrm{w}_{2}$ & & \\
\hline \multicolumn{8}{|c|}{ 2-Propanol/ $/ \mathrm{Na}_{2} \mathrm{SO}_{4} /$ Água } \\
\hline 0,2498 & 0,0998 & 0,3767 & 0,0252 & 0,0365 & 0,2252 & 0,3946 & $-1,7006$ \\
\hline 0,2698 & 0,1249 & 0,4852 & 0,0105 & 0,0173 & 0,2591 & 0,5298 & $-1,8820$ \\
\hline 0,2918 & 0,1495 & 0,5737 & 0,0044 & 0,0050 & 0,2971 & 0,6396 & $-1,9430$ \\
\hline \multicolumn{8}{|c|}{ 2-Propanol/ $\mathrm{NaCH}_{3} \mathrm{COO} /$ Água } \\
\hline 0,5597 & 0,1199 & 0,6924 & 0,0636 & 0,0577 & 0,3331 & 0,6895 & $-2,3550$ \\
\hline 0,5895 & 0,1143 & 0,7147 & 0,0617 & 0,0557 & 0,3415 & 0,7159 & $-2,3554$ \\
\hline 0,6189 & 0,1098 & 0,7347 & 0,0594 & 0,0384 & 0,3629 & 0,7596 & $-2,2943$ \\
\hline \multicolumn{8}{|c|}{ 2-Propanol/ $\mathrm{NaNO}_{3} /$ Água } \\
\hline 0,6133 & 0,1047 & 0,7323 & 0,0440 & 0,0239 & 0,4057 & 0,7954 & $-1,9585$ \\
\hline 0,5990 & 0,1198 & 0,7690 & 0,0340 & 0,0197 & 0,4121 & 0,8393 & $-1,9819$ \\
\hline 0,5590 & 0,1446 & 0,7856 & 0,0295 & 0,0132 & 0,4221 & 0,8665 & $-1,9672$ \\
\hline
\end{tabular}

Os valores de ILA para os sistemas estudados apresentaram o mesmo comportamento observado em outros estudos (Lo et al., 2015; Sanglard et al., 2018), onde o aumento das quantidades de 2-propanol e/ou sal resultou em valores mais altos de ILA. No sistema com sulfato, o aumento da ILA elevou os valores de CLA refletindo o comportamento encontrado no estudo de Pimentel et al. (2017). Por outro lado, os sistemas com acetato ilustraram um comportamento muito semelhante ao trabalho de Lo et al. (2015), marcado pelo aumento do CLA até certo ponto, seguido de uma redução. Quanto aos sistemas de nitrato, seus valores de CLA se comportaram de maneira similar aos observados nos sistemas de acetato. Informações sobre linhas de amarração de sistemas 2-propanol $+\mathrm{NaNO}_{3}+$ água não foram encontradas na literatura para fins de comparação.

Os valores, apresentados na Tabela 3, para os $\mathrm{R}^{2}$ e para os DP ilustram o bom ajuste e a precisão dos dados experimentais através das equações de Othmer-Tobias e Bancroft.

Tabela 3 - Parâmetros das Equações 5 e 6 para os sistemas 2-propanol/sais de sódio/água

\begin{tabular}{|c|c|c|c|c|c|c|c|c|}
\hline \multirow{2}{*}{ Sistema } & \multicolumn{4}{|c|}{ Othmer-Tobias } & \multicolumn{4}{|c|}{ Bancroft } \\
\hline & $\mathrm{k}_{1}$ & $\mathrm{n}$ & $\mathrm{R}^{2}$ & DP & $\mathrm{k}_{2}$ & $\mathrm{r}$ & $\mathrm{R}^{2}$ & DP \\
\hline 2-Propanol/ $\mathrm{Na}_{2} \mathrm{SO}_{4} /$ Água & 0,1160 & 2,1374 & 0,99 & 0,004 & 2,7047 & 0,4309 & 0,99 & 0,001 \\
\hline 2-Propanol/ $\mathrm{NaCH}_{3} \mathrm{COO} / \mathrm{Água}$ & 0,1556 & 1,4809 & 0,93 & 0,004 & 2,9323 & 0,4471 & 0,96 & 0,008 \\
\hline 2-Propanol/NaNO $/$ Água & 0,0728 & 4,1385 & 0,90 & 0,005 & 1,7437 & 0,1790 & 0,90 & 0,014 \\
\hline
\end{tabular}

\section{CONCLUSÕES}

Neste trabalho, os dados de equilíbrio líquido-líquido para os sistemas 2-propanol + $\mathrm{Na}_{2} \mathrm{SO}_{4} / \mathrm{NaNO}_{3} / \mathrm{NaCH}_{3} \mathrm{COO}$ + água foram obtidos experimentalmente a $298,15 \mathrm{~K} \mathrm{e} \mathrm{101,3} \mathrm{kPa.}$ Os dados das curvas binodais mostraram excelente correlação usando as equações de Merchuk et al. e Mistry et al. As equações de Othmer-Tobias e Bancroft provaram sua confiabilidade nos 


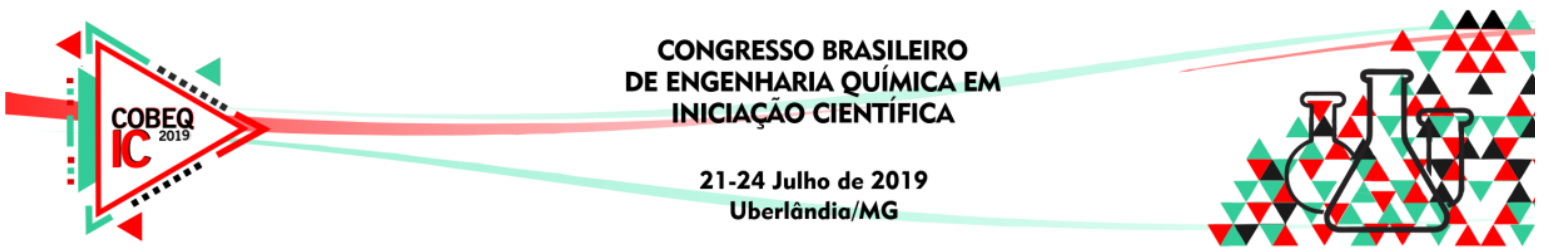

dados de linhas de amarração através dos valores de $\mathrm{R}^{2}$ e DP observados. Os sistemas de sulfato mostraram duas fases muito aquosas. No entanto, os sistemas de acetato e nitrato resultaram em uma fase rica em álcool e uma fase rica em sal. Este comportamento é interessante para uma separação entre o 2-propanol e a água usando uma extração líquido-líquido, futuramente. Os dados de composição das linhas de amarração dos sistemas com sulfato foram melhor correlacionados pela equação de Othmer-Tobias.

\section{AGRADECIMENTOS}

O presente trabalho foi realizado com apoio da Coordenação de Aperfeiçoamento de Pessoal de Nível Superior - Brasil (CAPES) - Código de Financiamento 001. Os autores agradecem, também a FAPERJ pelo apoio financeiro e ao CNPq-PIBIC pela bolsa de iniciação científica fornecida.

\section{REFERÊNCIAS BIBLIOGRÁFICAS}

DUTIA, P. Isopropyl alcohol: A techno-commercial profile, 2012.

GONZÁLEZ-TELLO, P.; CAMACHO, F.; BLÁZQUEZ, G.; ALARCÓN, F. J. Liquid-Liquid Equilibrium in the System Poly(ethylene glycol)+MgSO4+H2O at 298 K. J. Chem. Eng. Data, v. 41, p. 1333-1336, 1996.

LO, S. C.; RAMANAN, R. N.; TEY, B. T.; LING, T. C.; SHOW, P. L.; OOI, C. W. LiquidLiquid Equilibrium of Alcohols + Ammonium/Potassium/Sodium Acetate + Water Systems: Experimental and Correlation. J. Chem. Eng. Data, v. 60, p. 2848-2857, 2015.

MERCHUK, J. C.; ANDREWS, B. A.; ASENJO, J. A. Aqueous two-phase systems for protein separation: Studies on phase inversion. J. Chromatogr. B Biomed. Sci. Appl., v. 711, ed. 1-2, p. 285-293, 1998.

MISTRY, S. L.; KAUL, A.; MERCHUK, J. C.; ASENJO, J. A. Mathematical modelling and computer simulation of aqueous two phases continuous protein extraction. J. Chromatogr A., v. 741, ed. 2, p. 151-163, 1996.

OTHMER, D. F.; TOBIAS, P. E. Liquid-Liquid Extraction Data - The Line Correlation. Ind. Eng. Chem., v. 34, ed. 6, p. 693-696, 1942.

PIMENTEL, J. G.; BICALHO, S. F.; GANDOLFI, O. R. R.; VERISSIMO, L. A. A.; CASTRO, S. d. S.; SOUZA, E. A.; VELOSO, C. M.; FONTAN, R. d. C. I.; SAMPAIO, V. S.; Bonomo, R. C. F. Evaluation of salting-out effect in the liquid-liquid equilibrium of aqueous two-phase systems composed of 2-propanol and $\mathrm{Na} 2 \mathrm{SO} 4 / \mathrm{MgSO} 4$ at different temperatures. Fluid Phase Equilib., v. 450, p. 184-193, 2017.

SANGLARD, M. G.; FARIAS, F. O.; SOSA, F. H. B.; SANTOS, T. P. M. d.; IGARASHIMAFRA, L.; MAFRA, M. R. Measurement and correlation of aqueous biphasic systems composed of alcohol (1-propanol/2-propanol/tert-butanol)+(NH4)2SO4+ $\mathrm{H} 2 \mathrm{O}$ at $298 \mathrm{~K}$ and a textile dye partition. Fluid Phase Equilib., v. 466, p. 7-13, 2018. 